\title{
补偿列紧理论应用途径的研究
}

\author{
陈贵强 \\ (中国科学院系统科学研究所,北京) \\ 陆云光 \\ (中国科学院武汉数学物理研究所)
}

补偿列紧理论在偏微分方程中应用的研究已经取得了许多重要的结果 ${ }^{a-100}$, 但就我们所 知, 主要有两种应用途径: 一种是 Tartar ${ }^{\mathfrak{d} 1}$ 的 Young 测度静态结构分析法, 另一种则是 Diperna ${ }^{\text {[10] }}$ 的 Young 测度动态行为分析法。在这两种方法中都用到了Young 测度表示弱极 限定理. 最终目的是证明由逼近解序列所唯一确定的 Young 测度族均为 Dirac 测度. 但 这些方法都有它的间接性, 本文给出一条直接的应用途径来证明单个守恒律的柯西问题的逼 近解序列的收敛性,也就是问题

广义解的存在性.

$$
\left\{\begin{array}{l}
\frac{\partial u}{\partial t}+\frac{\partial f(u)}{\partial x}=0, t>0,-\infty<x<\infty \\
\left.u\right|_{t=0}=u_{0}(x)
\end{array}\right.
$$

在本文中, 我们仅用到了 $2 \times 2$ 行列式的弱连续定理而没有引进测度表示弱根限定理。

值得指出的是, Tartar 曾对 Hopf 方程 $\left(f(u)-\frac{1}{2} u^{2}\right)$ 也作过这方面的尝试.

首先, 我们得到如下紧性定理:

定理 1 (紧性框架) 设 $\varrho \subset R \times R^{+}$为有界开集, $\left\{u^{e}\right\}\left(u^{e}: \Omega \rightarrow R\right)$ 是一函数序列, 使 㧹

$$
u^{\mathrm{e}} \stackrel{*}{\longrightarrow} u,\left(L^{\infty}(\Omega)\right)(“ \stackrel{*}{\longrightarrow} \text { ”表示弱*收敛), }
$$

$f \in C^{\prime}\left[-M_{0}, M_{0}\right], M_{0}=\sup _{\bullet}\left\|u^{\bullet}\right\|_{L^{\infty}}$, 有

$$
f\left(u^{8}\right) \stackrel{*}{\longrightarrow} v,\left(L^{\infty}(0)\right) .
$$

且假定

$$
\eta_{i}\left(u^{a}\right)_{i}+q_{i}\left(u^{a}\right)_{x}(i-1,2 .) \text { 在 } H_{\text {loc }}^{-1}(\Omega) \text { 中紧, }
$$

这里 $\left(\eta_{1}(\lambda), q_{1}(\lambda)\right)=(\lambda-k, f(\lambda)-f(k))$,

那么 1) $v=f(u)$, a. e.

$$
\left(\eta_{2}(\lambda), q_{2}(\lambda)\right)=\left(f(\lambda)-f(k), \int_{k}^{2} f^{\prime 2}(s) d s\right), k \text { 是一个任意常数, }
$$

2) 如果没有区间使得 $f$ 是线性的, 那么 $u^{\boldsymbol{u}} \rightarrow u$, a.e.

注. 若 $f \in C^{2}\left[-M_{0}, M_{0}\right]$, 那么 2$)$ 的条件即为 $f^{\prime \prime}(u) \neq 0$, a. e. 于 $\left[-M_{0}, M_{0}\right]$.

证 利用 (3)式和 $2 \times 2$ 行列式弱连续定理知

本文1987年5月21日收到. 


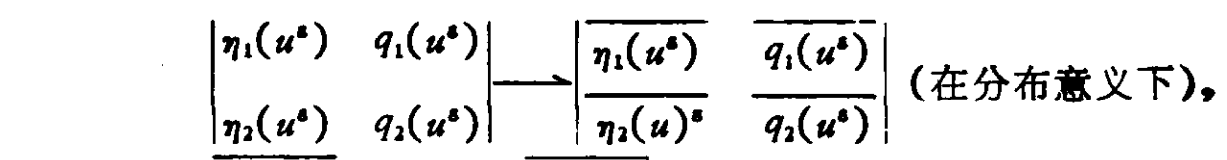

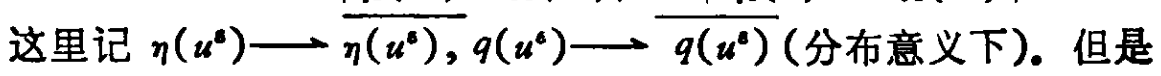

$$
\begin{aligned}
\left|\begin{array}{ll}
\eta_{1}\left(u^{a}\right) & q_{1}\left(u^{*}\right) \\
\eta_{2}\left(u^{a}\right) & q_{2}\left(\varepsilon^{a}\right)
\end{array}\right| & \\
& =\left(u^{a}-u\right) \int_{*}^{u^{*}} f^{\prime 2}(s) d s-\left(f\left(u^{a}\right)-f(u)\right)^{2} \\
& +(u-k) \int_{*}^{u^{*}} f^{\prime 2}(s) d s+\left(u^{*}-k\right) \int_{k}^{*} f^{\prime 2}(s) d s \\
& -(f(u)-f(k))^{2}-2\left(f\left(u^{a}\right)-f(u)(f(u)-f(k)),\right.
\end{aligned}
$$

并且

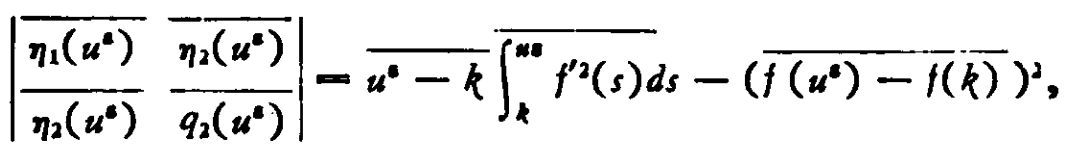

那么存在一个零测度集 $Q_{1}$, 对任给 $(x, t) \in Q_{1}^{c}$ 有

$$
\begin{aligned}
& \overline{\left(u^{*}-u\right) \int_{u}^{u^{*}} f^{\prime 2}(s) d s-\left(f\left(u^{2}\right)-f(u)\right)^{2}}+\overline{(u-k) \int_{u}^{u^{2}} f^{\prime 2}(s) d s} \\
& +\overline{\left(u^{a}-k\right) \int_{k}^{u} f^{\prime 2}(s) d s}-(f(u)-f(k))^{2} \\
& -2 \overline{\left(f\left(u^{*}\right)-f(u)\right)(f(u)-f(k))} \\
& -\overline{u^{0}-k} \int_{k}^{u^{a}} \overline{f^{\prime 2}(s) d s}-\overline{\left(f\left(u^{s}\right)-f(u)\right)^{2}} \\
& -2 \overline{\left(f\left(u^{a}\right)-f(u)\right)(f(u)-f(k))}-(f(u)-f(k))^{2} \text {. }
\end{aligned}
$$

因为

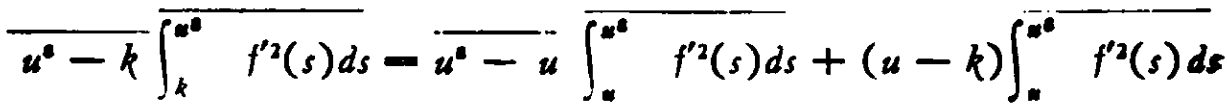

$$
\begin{aligned}
& +\overline{u^{2}-k} \int_{k}^{u} f^{\prime 2}(s) d s=(u-k) \overline{\int_{*}^{u t} f^{\prime 2}(s)} d s \\
& +\overline{\left(u^{2}-k\right)} \int_{k}^{\mu} f^{\prime 2}(s) d s \text {, }
\end{aligned}
$$

所以

$$
\overline{\left(u^{8}-u\right) \int_{u}^{u^{8}} f^{\prime 2}(s) d s-\left(f\left(u^{8}\right)-f(u)\right)^{2}}+\overline{\left(f\left(n^{8}\right)-f(u)\right)^{2}}=0 .
$$

因为

$$
\left(u^{2}-u\right) \int_{u}^{u^{8}} f^{\prime 2}(s) d s-\left(f\left(u^{8}\right)-f(u)\right)^{2} \geqslant 0,
$$

那么 $\overline{f\left(u^{\varepsilon}\right)}-f(u)=0$, 也就是 $v=f(u)$, a.e.

以及 $\overline{\left(u^{2}-u\right) \int_{u}^{u^{8}} f^{\prime 2}(s) d s-\left(f\left(u^{2}\right)-f(u)\right)^{2}}=0$, 所以

$$
\lim _{u \rightarrow 0} \int_{0}\left\{\left(u^{s}-u\right) \int_{*}^{u^{*}} f^{\prime 2}(s) d s-\left(f\left(u^{0}\right)-f(u)\right)^{2}\right\} d x d t=0 .
$$

642

科学 通报

1088

httrs://engine.scichina.com/doi/10.1360/csb1988-33-9-641 
对任賞常数 $\alpha>0$, 有

$$
\lim _{a \rightarrow 0} \int_{Q\left(\left|u^{s}-a\right|>\alpha\right)}\left\{\left(u^{e}-u\right) \int_{u}^{\omega^{e}} f^{\prime 2}(s) d s-\left(f\left(u^{0}\right)-f(u)\right)^{2}\right\} d x d t=0,
$$

因为

$$
\frac{d}{d \lambda}\left\{(\lambda-u) \int_{\alpha}^{\lambda} f^{\prime 2}(s) d s-(f(\lambda)-f(u))^{2}\right\}-\int_{0}^{\lambda}\left(f^{\prime}(\lambda)-f^{\prime}(s)\right)^{2} d s,
$$

那么如果没有区间使得 $f^{\prime \prime}=0$, 则

$$
\begin{gathered}
\int_{Q\left(\left(u^{a}-u\right)>a\right)}\left\{\left(u^{a}-u\right)^{u} \int_{u}^{u^{a}} f^{\prime 2}(s) d s-\left(f\left(u^{a}\right)-f(u)\right)^{2}\right\} d x d t \\
\geqslant C_{a} \operatorname{mes}\left(Q\left(\left(u^{s}-u\right)>a\right)\right) .
\end{gathered}
$$

同样

$$
\begin{gathered}
\int_{Q\left(\left(u^{\varepsilon}-u\right)<-\alpha\right)}\left\{\left(u^{\varepsilon}-u\right) \int_{u}^{x^{\varepsilon}} f^{\prime 2}(s) d s-\left(f\left(u^{\varepsilon}\right)-f(u)\right)^{2}\right\} d x d t \\
\geqslant C_{\alpha} \operatorname{mes}\left(s\left(\left(u^{e}-u\right)<-\alpha\right)\right),
\end{gathered}
$$

所以, 对任意常数 $\alpha>0$, 有

$$
\lim _{c \rightarrow 0} \operatorname{mes}\left(\Omega\left(\left|u^{8}-u\right|>\alpha\right)\right)=0,
$$

那么存在子序列 $u^{8 k}$ 使得 $u^{8 k} \rightarrow u$, a.e. 0 .

对于紧性框架(2)和(3), 有

定理 2 假定 $u_{0}(x) \in w^{1, \infty}, f \in C^{2}\left(-\left\|u_{0}(x)\right\|_{L^{-}},\left\|u_{0}(x)\right\|_{L^{\infty}}\right)$, 那么由下面柯西问题:

$$
\left\{\begin{array}{l}
\frac{\partial u^{\natural}}{\partial t}=\frac{\partial f\left(u^{\mathrm{e}}\right)}{\partial x}=\varepsilon \frac{\partial^{2} u^{\mathrm{e}}}{\partial x^{2}}, \\
\left.u^{\mathrm{s}}\right|_{\imath-\infty}-u_{\varepsilon}^{\mathrm{a}}(x) \equiv \int_{-\infty}^{\infty} i_{\varepsilon}(x-y) u_{0}(y) d y, i_{\bullet}(x) \text { 是一磨光算子, }
\end{array}\right.
$$

所唯一定义的粘性逼近解满足(2)和(3)式.

这个定理的证明见文献 [1]。

定理 3 对一状态 $\bar{u}, u_{0}(x)-\bar{u} \in L_{\infty} \cap L^{2}(-\infty, \infty), f \in C^{2}\left[-\left\|u_{0}(x)\right\|_{L^{\infty}},\left\|u_{0}(x)\right\|_{L^{\infty}}\right]$, 那么关于柯西问题 (1) 的 Lax-Fridrichs 格式和 Godunov 格式的逼近解满足紧性框架 (2) 和 (3).

这个定理的证明见文献 [6-7].

由定理 2 和定理 3, 我们有下面的存在性定理。

定理 4 如果 $u_{0}(x) \in w^{1, \infty}(-\infty, \infty)$ 或 $u_{0}(x)-\bar{u} \in L^{\infty} \cap L^{2}(-\infty, \infty) f \in C^{2}\left(-\| u_{0}(x)\right.$ $\left.\left\|_{L^{\infty}}\right\| u_{0}(x) \|_{L^{\infty}}\right)$ ，那么柯西问题 (1) 有一广义解 $u \in L^{\infty}$, 也就是

$$
\int_{0}^{\infty} \int_{\infty}^{\infty}\left\{\eta(u) \varphi_{t}+\eta(u) \varphi_{x}\right\} d x d t \geqslant 0,
$$

这里 $C^{2}$ 函数对 $(\eta, q)$ 满足 $q^{\prime}=\eta^{\prime} f^{\prime}, \eta^{\prime \prime}>0 . \quad \varphi \in C_{0}^{\infty}\left(k \times k^{+}-\{t=0\}\right)$, 是一个正函 数。

注。把直接方法这一思想用于拟线性双曲型方程组整体间断解存在性的研究，选取几对 有代表意义的摘一摘流对, 并借助于补偿列紧理论的一些基本定理, 而达到所期望的目标, 是一 个十分有意义的事情,值得更深入的探索。 
致谢：承蒙我们的老师丁夏畦教授的指导,在此表示谢意。

\section{考文 虾}

[1] Tartar, T., Nonlinear Analysis and mechanics, Research Notes in Mashemarics, Heriot-watt Symposium, Vol. 4. (Ed. Rnops, R. J.) Pitman Press, New York, 1979.

[2] Murat, F., Ann. Scuole Norm. Sup. Pisa Sci. Pis. Math, 5(1978), 489-507.

[3] Dacorogna B., Lectures Nozes in Math, Vol. 922, Springer, 1982.

[ 4] Diperna, R. J., Arch. Rat. Mech. Anal, 82(1983), 27-70.

[ 5 ] Comm. Mash. Phys, 9(1983), 1-30.

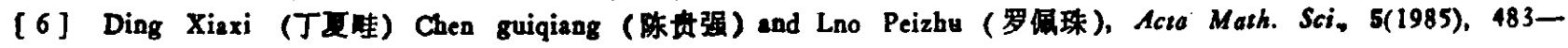
500, 501-540.

[7] Chen Guiqiang (陈贵强), Acra Mash. Sci, 6(1986), 75-120.

[ 8 ] Diperna, K. and Majda, A., Oscillations and concertrations in weak solutions of the incompresible fluid equations, Preprint (Submitted to Comm. Math. Phy.),

[ 9 ] Morawetz, C. S., Comm. Pure, Appl. Math, 38(1985), 797-818.

[10] Diperne, R. J., Arck. Ree. Mech. Amal, 88(1985), 223-270. 